\title{
Técnicas de imagen en la coartación de aorta
}

\author{
Amadeo Wals-Rodriguez \\ Maria Jose Rodriguez-Puras \\ Pastora Gallego
}

\begin{tabular}{l} 
Correspondencia \\
\hline Pastora Gallego \\
antoniap.gallego.sspa@juntadeandalucia.es \\
\hline
\end{tabular}

Unidad de Cardiopatías Congénitas del Adulto. Servicio de Cardiología. Hospital General Universitario Virgen del Rocío. Sevilla. Andalucía. España

Recibido:12/01/2019

Aceptado: 03/01/2020

En línea: 02/03/2020

Citar como: Wals-Rodriguez A, Rodriguez-Puras MJ, Gallego P. Técnicas de imagen en la coartación de aorta. Rev Ecocar Pract (RETIC). 2020 (Mar); 3 (1): 7-13. doi: 10.37615/retic.v3n1a3.

Cite this as: Wals-Rodriguez A, Rodriguez-Puras MJ, Gallego P. Imaging techniques in aortic coarctation. Rev Ecocar Pract (RETIC). 2020 (Mar); 3 (1): 7-13. doi: 10.37615/retic.v3n1a3.

\section{Palabras clave \\ $\triangleright$ Coartación de aorta \\ $\triangleright$ Ecocardiografía \\ $\triangleright$ Resonancia magnética \\ $\triangleright$ Tomografía computarizada}

\section{RESUMEN}

La coartación aórtica es una malformación congénita relativamente frecuente. Sus manifestaciones abarcan un amplio espectro de presentación, pudiendo mostrar síntomas desde el nacimiento, o bien diagnosticarse en la edad adulta. Para su manejo se dispone de una amplia gama de técnicas de imagen que permiten valorar la anatomía de la estenosis, las repercusiones estructurales en el ventrículo izquierdo, las alteraciones hemodinámicas y las complicaciones asociadas. La ecocardiografía transtorácica es la técnica de primera línea. Para la valoración quirúrgica en la coartación nativa y el seguimiento de la coartación reparada, la tomografía computarizada y la resonancia magnética son las técnicas de elección.

ABSTRACT
Coarctation of aorta is a congenital malformation relatively frequent in the population. The clinical presentation
varies widely, from symptoms at birth to incidental diagnosis in adulthood. Imaging techniques allow us to assess
the anatomy of the stenosis, hemodynamics, left ventricle function and hypertrophy and the presence of asso-
ciated complications. Transthoracic echocardiography is the first line technique but computed tomography and
cardiac magnetic resonance are the tests of choice for surgical evaluation of the native coarctation and to follow-
up the repaired coarctation.

\section{Introducción}

La coartación de aorta (CoAo) es una malformación congénita consistente en un estrechamiento localizado de la luz del vaso. La constricción de la aorta se localiza a cualquier nivel, aunque es más frecuente en la región yuxtaductal|(1) (Figura 1). Corresponde aproximadamente al 5-8\% de todas las cardiopatías congénitas y se presenta en el $85 \%$ de los casos junto a lesiones asociadas, o formando parte de un síndrome establecido.

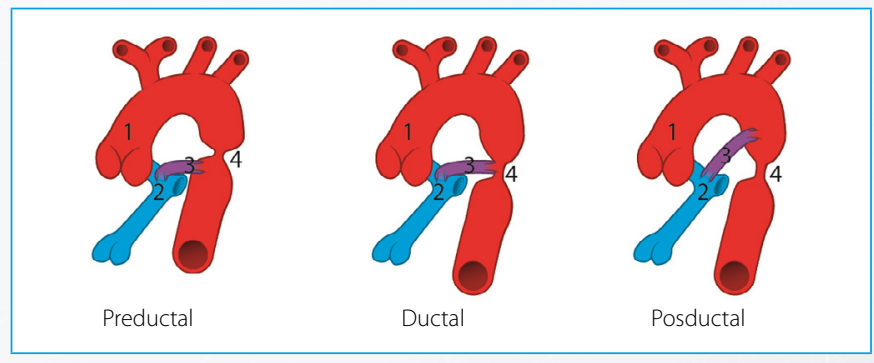

Figura 1. Clasificación anatómica de la coartación de aorta (1: aorta; 2: arteria pulmonar; 3: ductus arterioso; 4: coartación)
El diagnóstico se realiza en la mayoría de las ocasiones en la infancia. Sin embargo, es posible que la CoAo se presente de forma larvada, y su evolución se lleve a cabo durante años, hasta finalmente diagnosticarse en la edad adulta (Tabla 1).

\begin{tabular}{|l|l|}
\hline \multicolumn{1}{|c|}{\begin{tabular}{c}
\multicolumn{1}{c|}{$\begin{array}{c}\text { Clínica de la coartación } \\
\text { de aorta en el adulto }\end{array}$} \\
$\begin{array}{l}\text { Hipertensión arterial en el } \\
\text { hemicuerpo superior }\end{array}$
\end{tabular}} & \multicolumn{1}{c|}{ Índice tobillo-brazo aumentado } \\
\hline $\begin{array}{l}\text { Frialdad de miembros inferiores, } \\
\text { claudicación }\end{array}$ & $\begin{array}{l}\text { Pulso femoral disminuido } \\
\text { y retardado. Cambios tróficos } \\
\text { en miembros inferiores }\end{array}$ \\
\hline $\begin{array}{l}\text { Cefalea, epistaxis, hemorragia } \\
\text { intracraneal (en las formas que } \\
\text { asocian aneurismas del polígono de } \\
\text { Willis), insuficiencia cardíaca }\end{array}$ & $\begin{array}{l}\text { Soplo mesosistólico. En casos de } \\
\text { estenosis importante, es posible } \\
\text { que se produzca un soplo continuo } \\
\text { por un flujo de sangre de alta } \\
\text { velocidad mantenido todo } \\
\text { el ciclo }\end{array}$ \\
\hline
\end{tabular}

Tabla 1. Clínica y exploración física de la coartación aórtica en el adulto 


\section{Reparación quirúrgica. Técnicas}

Desde el inicio de la década de 1940 se han desarrollado varias técnicas quirúrgicas para el tratamiento de la CoAo, siendo las más frecuentes la anastomosis término-terminal tras coartectomía, la aortoplastia con flap de arteria subclavia o con parche de Dacron ${ }^{\circ}$. Injertos tubulares, cirugías complejas del arco aórtico y bypass extraanatómico son otras opciones (Figura 2). Todas las técnicas quirúrgicas tienen ventajas y desventajas y pueden presentar complicaciones, requiriendo estrecho seguimiento a largo plazo. Suele preferirse la cirugía reparadora en la infancia, y la reparación endovascular en la edad adulta.

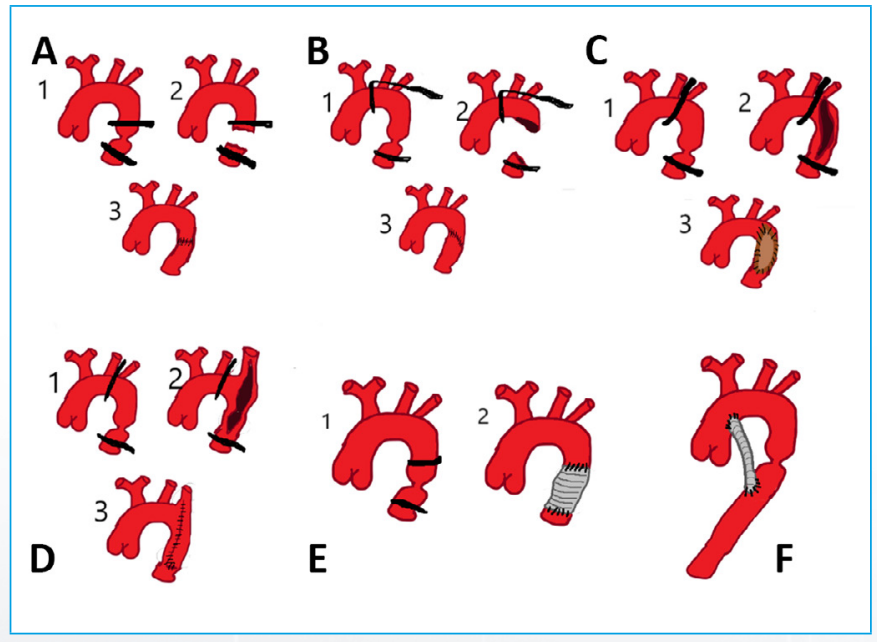

Figura 2. Ilustración de los diferentes tipos de cirugía en la coartación deaorta. A: resección y anastomosis término-terminal; B: resección y anastomosis término-terminal extendida; C: aortoplastia con parche protésico; D: aortoplastia con flap de subclavia; E: resección e interposición de conducto; F: interposición de conducto extraanatómico entre aorta ascendente y descendente. En todas las figuras los números se refieren a las fases del procedimiento

\section{Esperanza de vida y complicaciones de la coartación reparada}

La historia natural de la CoAo ha cambiado sustancialmente con la reparación en la infancia. Los resultados a corto plazo son excelentes, resultando en una excelente hemodinámica y en el alivio de la hipertensión arterial con una mortalidad precoz inferior al 2\%. La mayoría de los pacientes alcanzan la edad adulta. No obstante, los estudios de historia posquirúrgica publicados han llevado a que la CoAo deje de considerarse una obstrucción focal del istmo y se considere parte del espectro de una arteriopatía difusa. Resuelta la obstrucción mecánica, el problema fisiopatológico persiste y ocurren complicaciones en otras áreas del sistema arterial que tienen un enorme impacto en la morbimortalidad durante la vida adulta. A continuación, se exponen las complicaciones más frecuentes ${ }^{(2)}$ :

- Lesiones valvulares asociadas. Principalmente valvulopatía aórtica, entre las que destacan la válvula aórtica bicúspide (VAB) y la estenosis aórtica valvular o subvalvular. Frecuente también la valvulopatía mitral, en el síndrome de Shone, que asocia CoAo, estenosis mitral supravalvular y válvula mitral en paracaídas. Son las complicaciones más frecuentes, presentes en torno al $85 \%$ de los casos.

- Complicaciones de la pared arterial. El desarrollo de aneurismas de la aorta descendente (Figura 3) se relaciona con un aumento de la mortalidad, y está relacionado con la presencia de otros factores general- mente asociados a la CoAo, como sor! la VAB y la HTA. La prevalencia de complicaciones de la pared arterial en la población adulta con CoAo es de aproximadamente un 16\% y se incluyen aneurismas, roturas de aneurismas, disección, pseudoaneurisma, aneurismas micóticos y fístulas traqueobronquiales y entre cavidades $\operatorname{cardíacas}^{(3)}$. Dada la frecuencia de las complicaciones de la pared arterial y el pronóstico infausto que presentan, es muy importante realizar periódicamente técnicas de imagen que evalúen la totalidad de la aorta, con objeto de detectarlas y tratarlas de forma precoz.

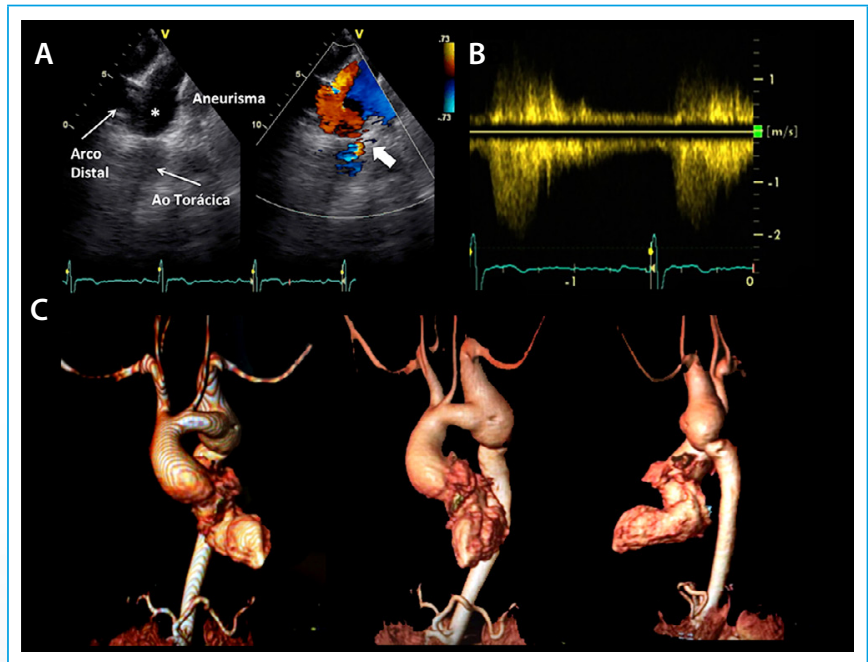

Figura 3. A: ecocardiografía transtorácica. Imagen bidimensional y con Doppler color desde proyección suprasternal de adulto con coartación reparada quirúrgicamente en la infancia y aneurisma en la región de la aortoplastia; B: el registro Doppler sugiere recoartación; C: estudio de angio-RM. Reconstrucción volumétrica de la aorta. Se aprecia dilatación de la aorta ascendente, con arco transverso de dimensiones normales y con un aneurisma entre el tercer arco aórtico y el istmo. Existe recoartación ligera

- Recoartación. Se define como una estenosis residual o recurrente hemodinámicamente significativa, después de la reparación (Figura 4), que induce o agrava la hipertensión arterial. Aparece en torno al 15\% de los pacientes. Se considera recoartación significativa cuando existe un gradiente transcoartación $>20 \mathrm{mmHg}^{(4)}$. Puede ocurrir independientemente de la técnica quirúrgica empleada en la corrección y la reparación a edad temprana se considera un factor de riesgo para su aparición. Debe descartarse en aquellos pacientes con CoAo reparada en los que aparezca nuevamente hipertensión arterial, o mal control en aquellos que presentaron hipertensión residual.

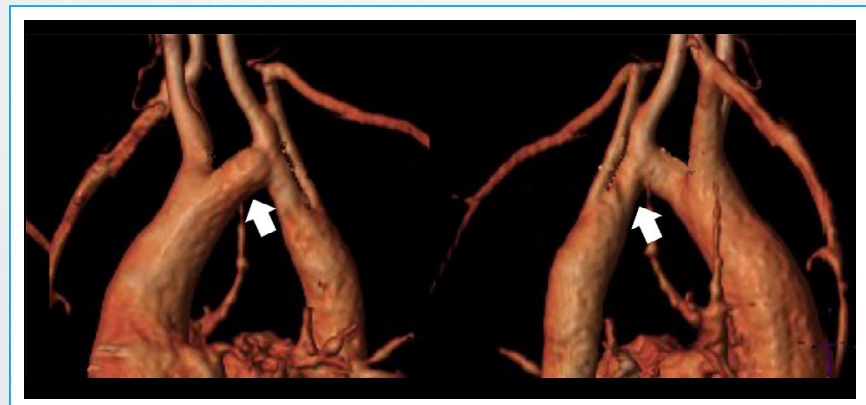

Figura 4. Angio-RM con reconstrucción tridimensional en varón de 17 años. Coartación reparada en la infancia mediante resección y anastomosis término-terminal. HTA sistólica e hipoplasia del segundo segmento del arco transverso (flecha). Se documenta un gradiente hemodinámico de $30 \mathrm{mmHg}$ 
- Hipertensión arterial (HTA). Constituye una complicación frecuente (aproximadamente el $27 \%$ de los pacientes con CoAo reparada) y se relaciona con un aumento de la morbimortalidad cardiovascular en los pacientes con CoAo debido al desarrollo precoz de cardiopatía hipertensiva y cardiopatía isquémica.

- Hipertensión pulmonar (HTP). Complicación tardía, pero con gran importancia en el pronóstico. Se debe sospechar la aparición de HTP en aquellos pacientes que en los estudios funcionales se evidencie disfunción diastólica del ventrículo izquierdo. Presente en el 18\% de los pacientes.

- Aneurismas intracraneales. Por su gran morbimortalidad asociada, tienen gran relevancia los que afectan al polígono arterial de Willis. La asociación de ambas entidades se conoce desde $1871^{(5)}$ y actualmente se sabe que el riesgo relativo para desarrollar aneurismas cerebrales es significativamente mayor en los pacientes de CoAo que en la población general(6) Están presentes en un 3-10\% de los pacientes, y no guardan relación ni con la edad, sexo, presión arterial, reparación, presencia de factores de riesgo cardiovasculares o neurológicos, o presencia de VAB.

\section{Objetivos de las técnicas de imagen}

La evaluación de la coartación de aorta se realiza integrando datos clínicos, anatómicos y hemodinámicos. Las técnicas de imagen ofrecen información no invasiva sobre estos dos últimos y son indispensables en la toma de decisiones clínicas:

- En la coartación nativa: confirmar el diagnóstico, evaluar la gravedad de la obstrucción y la anatomía de la coartación, identificar las lesiones anatómicas asociadas y las complicaciones secundarias a la obstrucción a la salida del ventrículo izquierdo (hipertrofia, disfunción sistólica y diastólica e hipertensión pulmonar)

- En el seguimiento de la coartación reparada: diagnóstico de recoartación o aneurismas de la aorta ascendente o torácica, la evolución de las lesiones asociadas y las complicaciones de la hipertensión arterial.

\section{Radiografía simple de tórax}

La radiografía simple fue la primera técnica de imagen que se describió y empleó en CoAo, y aunque no permite llegar a un diagnóstico de certeza, sí que es posible encontrar signos radiológicos, como son el signo de Rossler (muescas costales, producidas por el aumento de la circulación colateral torácica) y el signo del 3 (escotadura aórtica a nivel de la coartación y dilatación preestenosis y postestenosis). En caso de realizar un esofagograma con bario es posible visualizar el signo de la E, correspondiente a la curvatura del esófago alrededor de los segmentos preestenóticos y postestenóticos de la aorta. Actualmente la radiografía simple de tórax está incluida dentro de la valoración inicial de los pacientes, aunque su utilidad posterior es escasa ${ }^{(1)}$.

\section{Ecocardiografía transtorácica}

La ecocardiografía transtorácica (ETT) es muy útil en el diagnóstico y seguimiento de la coartación nativa y reparada(1). Se considera una técnica de primera línea en el diagnóstico y seguimiento de las cardiopatías congénitas y es posible adquirir imágenes anatómicas y funcionales.

- Ecocardiografía bidimensional. La ETT bidimensional permite obtener imágenes anatómicas de la coartación, valorar la función y la hipertrofia del ventrículo izquierdo, diagnosticar otras malformaciones intracardíacas asociadas y, en edades pediátricas, incluso medir los diámetros preestenosis y postestenosis de la aorta (Figura 5A). En el paciente adulto, las limitaciones de la ventana ecogénica hacen más difícil valorar la anatomía del arco aórtico, sobre todo en casos de coartación compleja o postoperada.

- Doppler continuo. El estudio Doppler consigue un aumento significativo de la sensibilidad de la ecocardiografía en la evaluación de la severidad de la coartación de aorta(7). El Doppler proporciona el gradiente máximo y medio transcoartación, aunque el signo más específico de severidad es la prolongación diastólica de la onda de flujo a nivel, de forma que permite cuantificar la gravedad de la estenosis (Figura 5B).

El Doppler continuo es preferible al Doppler pulsado. No obstante, son varias las limitaciones a la hora de obtener un gradiente máximo transcoartación fiables. El gradiente por Doppler infraestima la severidad en presencia de colaterales, pero en la mayor parte de los casos, al tratarse de una estenosis tubular y de una arteria más rígida, la velocidad sistólica máxima puede estar sobreestimada por el fenómeno de recuperación de presiones. Aunque el gradiente sistólico transcoartación es poco específico, la prolongación diastólica del flujo anterógrado de sangre a través de la coartación supone el predictor más importante cuando se emplea el Doppler continuo.

Por otra parte, el gradiente Doppler en el caso de una coartación reparada suele sobrevalorar la estenosis residual $\left.\right|^{(7)}$. Antes de la intervención la obstrucción tiene una morfología similar a un diafragma con dilatación brusca de la aorta descendente postístmica, mientras que después de la intervención, la obstrucción residual suele ser de morfología tubular, produciendo fenómenos hidrodinámicos de recuperación de presiones que amortiguan el gradiente invasivo, pero no así el obtenido por Doppler.

Doppler pulsado. El Doppler pulsado también tiene su aplicación en la valoración de la CoAo. El hallazgo más significativo con el modo pulsado es un retraso de la onda de pulso y una prolongación del flujo anterógrado en diástole en la aorta abdominal (Figura 5C).

- Doppler color. El empleo del Doppler color permite identificar aumentos de la velocidad del flujo en la zona de la coartación (Figura 5A), así como valorar la presencia de otras malformaciones como el ductus arterioso persistente o comunicaciones anómalas entre cavidades.

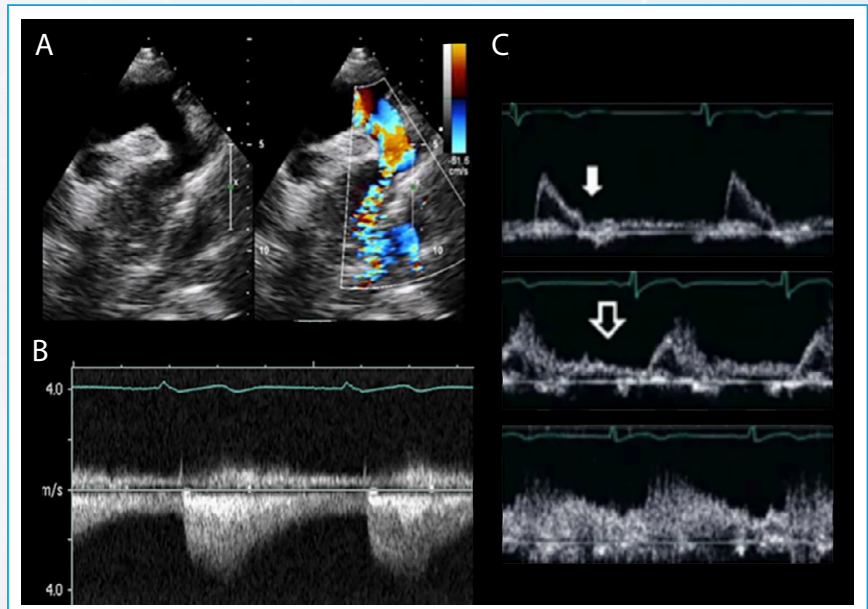

Figura 5. Ecocardiografía transtorácica. A: imagen bidimensional y con Doppler color desde proyección supraesternal en un paciente adulto con una coartación nativa. Estenosis circunscrita aórtica inmediatamente después de la salida de la arteria subclavia izquierda con dilatación postestenótica. La imagen permite medir las dimensiones de la aorta ascendente, el arco transverso proximal y distal, el istmo aórtico y la aorta torácica descendente. Con Doppler color se observa un flujo turbulento con zona de aceleración proximal en el istmo; B: patrón ecocardiográfico en modo Doppler: importante aceleración telesistólica del flujo (hasta 4 m/s) con prolongación diastólica característica; C: flujo con Doppler pulsado en aorta abdominal continuo, de baja velocidad, con pico máximo retrasado, característico de la coartación de aorta) 
- Diagnóstico de complicaciones. La ecocardiografía permite diagnosticar o sospechar complicaciones asociadas a la CoAo, como son la disfunción sistólica o diastólica del ventrículo izquierdo, la hipertrofia ventricular, presencia de HTP, valorar la aorta ascendente y el estado de las válvulas aórtica y mitral. Hallazgos sugestivos de complicaciones en el arco aórtico en el estudio ecocardiográfico, pueden justificar la realización de otras técnicas de imagen.

\section{Limitaciones de la ecocardiografía Doppler}

- Alto flujo: depende del gasto cardíaco y de la existencia de obstrucción en el tracto de salida del ventrículo izquierdo

- Aumento de la coartación: grado de tortuosidad

- Circulación colateral: condiciona un flujo bajo

Tabla 2. Limitaciones de la ecocardiografía Doppler para cuantificar el gradiente transcoartación

\section{Resonancia magnética cardíaca}

La resonancia magnética cardíaca (cardio-RM) y la tomografía computarizada (TC) son las técnicas de imagen no invasivas de elección para evaluar toda la aorta en adultos. Aportan información sobre la morfología del arco y de la coartación, el grado de estenosis, la longitud, la implicación de vasos supraaórticos, la presencia de colaterales, etc. Son fundamentales para confirmar el diagnóstico, para planificar cualquier tipo de intervención y para el seguimiento y diagnóstico de posibles complicaciones de la pared como aneurismas, reestenosis, etc. ${ }^{(8)}$.

La cardio-RM se ha convertido en la actualidad en una herramienta muy útil en el manejo de la CoAo, de tal modo que es una parte esencial de la evaluación rutinaria en el seguimiento de la CoAo reparada. Se considera hoy en día la técnica de imagen de referencia para el periodo postratamiento, a pesar de que las mediciones realizadas en el área de estenosis pueden no tener una correspondencia 100\% concordante con las mediciones realizadas durante la cirugía ${ }^{(9)}$.

\section{Ventajas y desventajas}

La cardio-RM ofrece grandes ventajas frente a otras técnicas de imagen. Permite realizar un estudio anatómico y funcional completo, sin limitaciones por la ventana acústica. Con respecto al estudio mediante angio-TC ofrece la ventaja de no utilizar radiación ionizante (los pacientes precisarán múltiples exploraciones a lo largo de su vida) y la posibilidad de realizar no sólo estudios angiográficos sino también exploraciones dinámicas de los flujos en la aorta. La cardio-RM es la prueba de referencia para la cuantificación de la fracción de eyección del ventrículo izquierdo, y además ofrece datos precisos sobre el grado de hipertrofia y fibrosis miocárdica mediante el empleo de técnicas de realce tardío y técnicas $\mathrm{T1}$ mapping.

Tiene como principal desventaja su coste y escasa disponibilidad, ya que se trata de una técnica compleja y que requiere equipamiento y profesionales altamente especializados para su realización e interpretación.

\section{Protocolo de realización}

Un protocolo de RM debe incluir:

- Secuencias de contraste de fase. Adquiridas a través del plano (Figura 6), en apnea con sincronización retrospectiva y con reconstrucción segmentada obteniendo una resolución temporal de 30 fases por ciclo cardíaco. Se utiliza un límite de velocidad estándar $(200 \mathrm{~cm} / \mathrm{s})$ y si la velocidad en el vaso supera este límite y aparece aliasing, se repite de nuevo la secuencia con velocidades progresivamente crecientes aumentando en cada una de ellas $50 \mathrm{~cm} / \mathrm{s}$ el límite de velocidad hasta la desaparición del aliasing. Las secuencias se adquieren en cortes de $6 \mathrm{~mm}$ de grosor programados en un plano doble oblicuo con el objetivo de obtener planos completamente perpendiculares al flujo aórtico; el primero inmediatamente proximal a la zona de la coartación, el segundo inmediatamente distal a la zona de la coartación y el tercero en aorta descendente a nivel del diafragma. Se evalúa también el fenotipo valvular aórtico y la presencia de valvulopatía aórtica mediante 3 cortes consecutivos sin espacio entre ellos programados en un plano doble oblicuo paralelo al plano valvular aórtico.

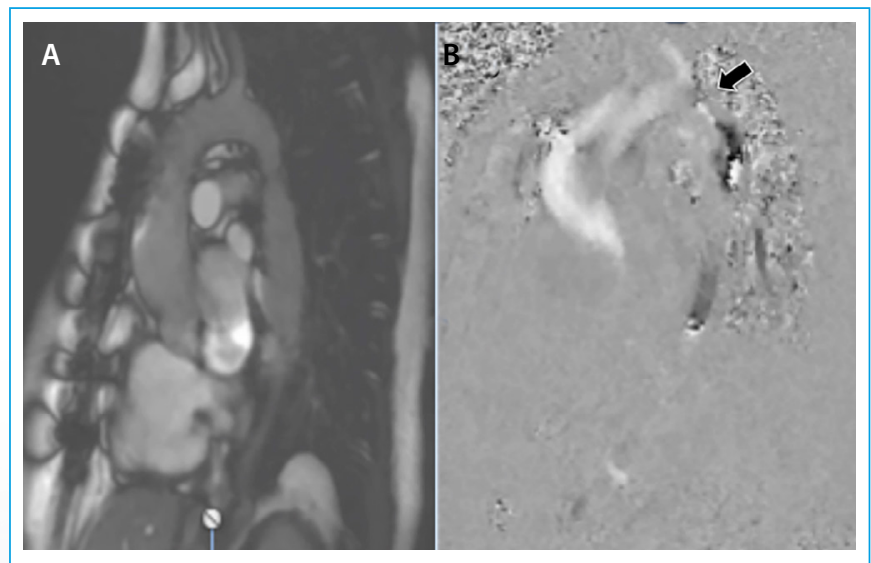

Figura 6. Secuencias de magnitud (A) y contraste de fase (B) obtenidas con resonancia magnética. Secuencia in plane para valorar cualitativamente el jet transcoartación (flecha negra) y así programar la secuencia through plane perpendicular al vaso con la que obtener datos cuantitativos

- Secuencias angiográficas. Permiten obtener numerosos cortes, de 1-1,5 mm de grosor en orientación coronal con la administración de gadolinio y seleccionando el momento de adquisición de la secuencia en función de la localización del contraste en las cavidades cardíacas.

\section{Procesado y análisis de imágenes}

En las secuencias de contraste de fase se valoran el volumen total de flujo inmediatamente proximal a la coartación; la velocidad y gradiente máximo poscoartación mediante la ecuación de Bernoulli y el volumen total de flujo en la aorta descendente a nivel del diafragma.

En las imágenes de la angiografía se realizan reconstrucciones multiplanares para obtener en un corte perpendicular a las paredes de la aorta y medir el diámetro y el área mínima en la zona de la coartación y el diámetro y área máxima en la aorta descendente a nivel del diafragma. Con la información obtenida se cuantifican la estenosis relativa (diámetro máximo de la aorta descendente a nivel del diafragma - diámetro mínimo en la zona de la coartación / diámetro máximo de aorta descendente x 100) y el incremento de flujo (flujo en aorta descendente diafragmática - flujo en aorta descendente proximal a la coartación / flujo en aorta proximal x 100).

\section{Cuándo realizar un estudio de resonancia magnética}

- Coartación nativa. Se recomienda una exploración diagnóstica inicial en la coartación nativa como parte del estudio de evaluación y planificación del tratamiento (Figura 7).

- Coartación reparada. La cardio-RM ha demostrado ser la técnica más coste-eficaz en el seguimiento de las complicaciones de la coartación reparada. Los pacientes con CoAo deben tener un estrecho seguimiento 
por cardio-RM independientemente del seguimiento ecocardiográfico. En nuestro medio se recomienda que a los pacientes con CoAo reparada se les realice una exploración cada 5-6 años. El control por cardio-RM puede adelantarse en el caso de que exista la sospecha de complicaciones basadas en la exploración física y ecocardiográfica(8) (véase la Figura 3).

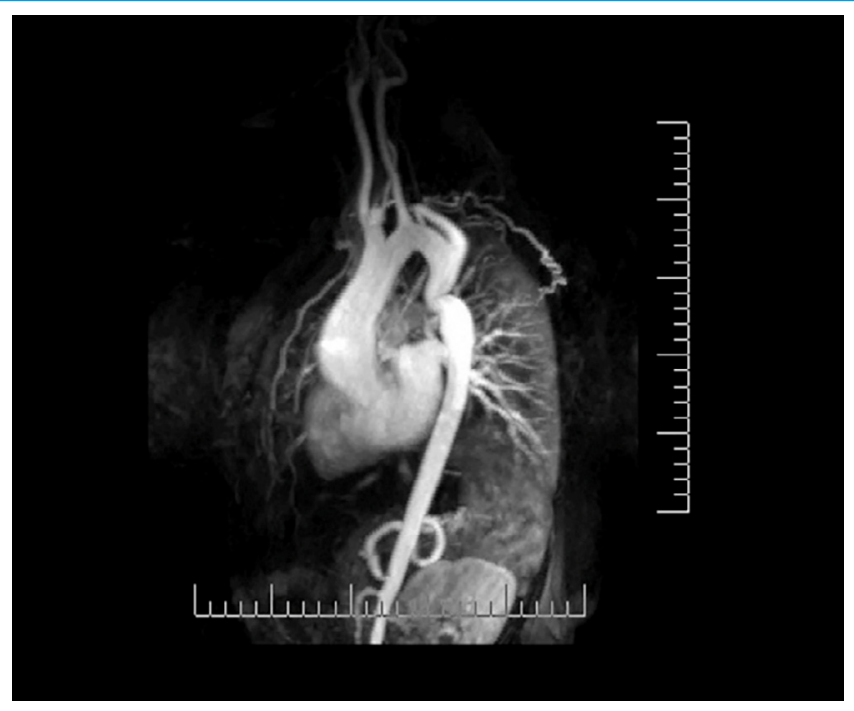

Figura 7. Angio-RM con reconstrucción MIP. Dilatación de aorta ascendente. Coartación aórtica yuxtaductal

\section{Qué información se debe recoger ${ }^{(9)}$}

Anatómica. Se debe localizar la zona de estenosis y cuantificar el porcentaje de la luz ocluida. Según dicho porcentaje es posible establecer la ausencia de estenosis si dicha oclusión es menor al 10\%. Se hablaría de estenosis moderada en aquellos casos en los que se cuantifique entre un 50-70\%, y de estenosis severa cuando el grado de oclusión supere el 70\%. Es importante, además, visualizar la relación de la lesión con los grandes vasos, valorar la presencia de circulación colateral (Figura 8) (la angio-TC ha demostrado ser superior que la cardio-RM en este caso), estudiar adecuadamente la válvula aórtica, y evaluar el ventrículo izquierdo, midiendo su función, grado de hipertrofia, el carácter de la hipertrofia, y el grado de fibrosis miocárdica.

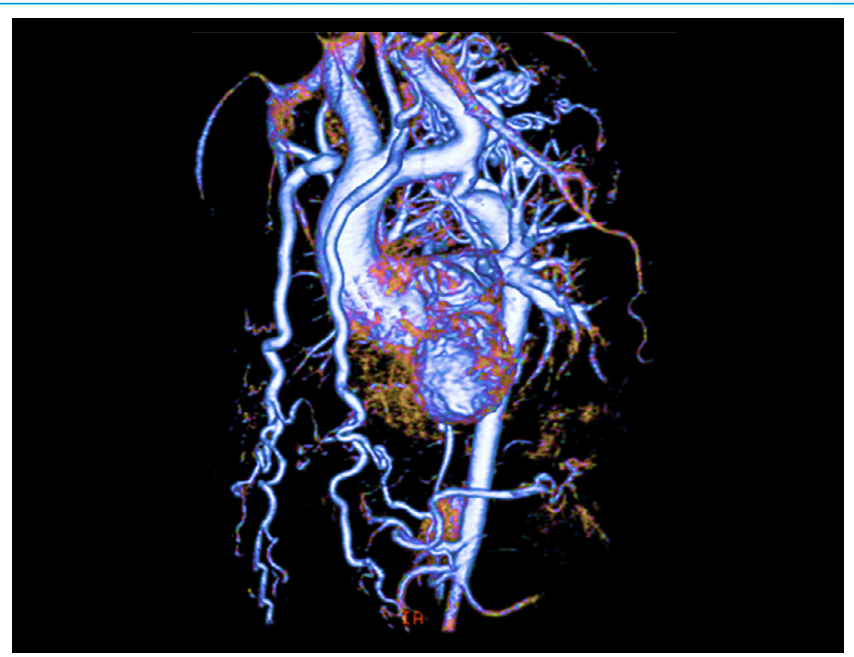

Figura 8. Angio-RM con reconstrucción de superficie. Coartación aórtica yuxtaductal severa. Importante flujo colateral incluyendo hipertrofia de arterias mamarias internas
Hemodinámica. Estimación del gradiente transcoartación (mediante la fórmula de Bernouilli). Se considera significativo si es $>20 \mathrm{mmHg}$.

\section{Tomografía computarizada}

Es la modalidad de imagen de primera elección para el diagnóstico y caracterización de la enfermedad aórtica en adultos y la técnica alternativa para niños en los que no pueden obtenerse imágenes de RM de suficiente resolución. Esta técnica estudia la aorta de una manera no invasiva, rápida y con gran detaIle. En las cardiopatías congénitas complejas conocidas o sospechadas, la TC se puede emplear para obtener imágenes del arco aórtico y de los grandes vasos para la evaluación postoperatoria, facilitando una valoración rápida y completa de las estructuras cardiovasculares o la detección de complicaciones postratamiento. La combinación de imágenes axiales, reformateos en dos dimensiones y MIP, y reconstrucciones tridimensionales (Figura 9) permite definir las relaciones anatómicas ${ }^{(11)}$

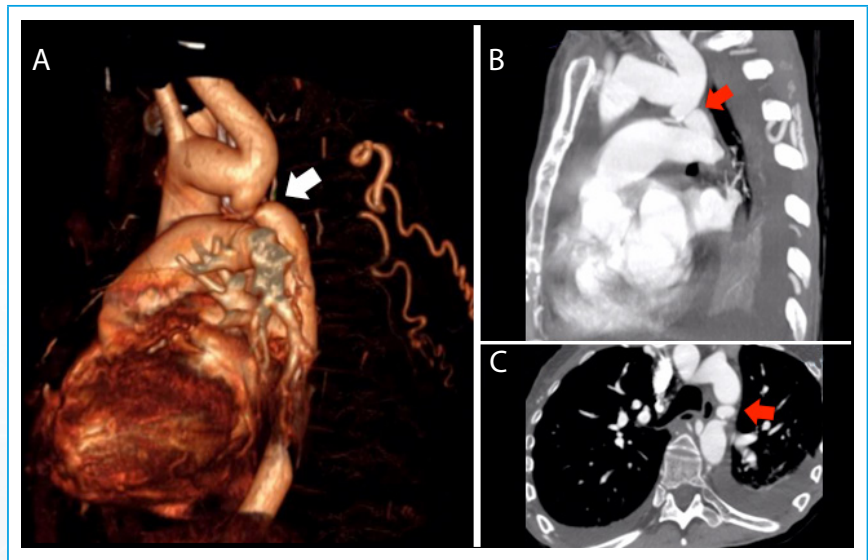

Figura 9. Angio-TC multidetector en adulto con coartación nativa posductal. El estrechamiento (flechas) se presenta distal a la arteria subclavia izquierda. Existe un segmento corto de la aorta yuxtaductal disminuido de calibre, asociado a dilatación aórtica proximal y distal a la estenosis y a formación de circulación colateral (arterias intercostales, torácicas internas, etc.). A: reconstrucción tridimensional; B: reconstrucción multiplanar oblicua; C: imagen axial

\section{Ventajas y desventajas}

Es cierto que la cardio-RM se ha convertido prácticamente en la prueba de imagen estrella en la CoAo, pero no es menos cierto que la TC actualmente es una herramienta casi indispensable en el diagnóstico y en el manejo de la CoAo, aportando ciertas ventajas respecto a la anterior, como son la posibilidad de realizarse en pacientes con implantes metálicos, y la baja tasa de artefactos en pacientes portadores de stents coronarios. Resulta una técnica muy precisa, sensible y específica, con una concordancia casi del $100 \%$ en algunas series respecto a la medición del diámetro de la coartación, comparada con la medición realizada en el quirófano.

Las principales ventajas de la TC se resumen en:

- Evaluación global, incluyendo pulmones, vías aéreas y otras estructuras regionales, afectados tanto de patologías adquiridas como congénitas.

- Análisis de las repercusiones de las alteraciones cardiovasculares en el sistema respiratorio, comprimiendo o desviando la tráquea (anillos vasculares), produciendo atrapamiento aéreo, hipoinsuflación, perfusión en mosaico, edema de pulmón, etc.

- Permite estudiar anomalías primarias del sistema respiratorio como hipoplasia pulmonar o traqueomalacia. 
- En ocasiones requiere sedación, pero raramente anestesia.

- Tiempo de examen reducido (menor que la resonancia) y habitualmente bien tolerado.

- No es operador dependiente (al contrario que la ecocardiografía).

- La capacidad multiplanar y tridimensional de la angio-TC multidetector permite obtener información en cualquier plano, a diferencia de la angiografía.

- Es una técnica accesible con menor coste que la RM o la angiografía.

Sin embargo, no hay que olvidar que es una técnica que emplea radiación ionizante, y que los pacientes con CoAo son pacientes que se diagnostican generalmente a edades tempranas y van a necesitar estudios de imagen cada pocos años durante toda su vida ${ }^{(1)}$. Además, emplea contraste yodado, aunque el riesgo de reacciones adversas mayores al contraste yodado (espasmo de las vías aéreas y colapso cardiovascular) es extremadamente bajo.

\section{Indicaciones}

- Diagnóstico anatómico en la coartación nativa. La zona de coartación y su extensión deben analizarse de la forma más precisa posible, y es importante describir la presencia de válvula aórtica bicúspide y sus características hemodinámicas. La angio-TC además permite el estudio de la circulación colateral, importante para la reparación. También es necesario el cribado de otras malformaciones asociadas. La TC ofrece la posibilidad de realizar reconstrucciones tridimensionales de la aorta (Figura 10), que también suponen herramientas muy útiles a la hora de planificar la reparación posterior $^{(1)}$.

- Valoración de complicaciones de la pared arterial en CoAo reparada. Las diferentes técnicas y modalidades de la TC han demostrado ser útiles para evaluar complicaciones posteriores a la colocación de stents para la corrección de la CoAo (Figura 10), como son el desarrollo de fracturas o movilizaciones del implante, trombosis, formación de aneurismas o disección ${ }^{(1)}$.

- Sospecha de enfermedad coronaria adquirida ${ }^{(12)}$. La TC permite obtener imágenes de las arterias coronarias con una calidad aceptable en un gran número de pacientes. Para el cribado de enfermedad ateroesclerótica es posible emplear técnicas sin contraste, como el conocido score de calcio o de Agatson, y técnicas con contraste, como la angio-TC coronaria. Actualmente no existe ningún protocolo específico, ni ningún consenso sobre cuándo y qué prueba realizar para detectar lesiones coronarias ateroescleróticas en los pacientes con CoAo. Por ello, las indicaciones para la realización de pruebas de detección de isquemia son las mismas que se aplican en la población general. La enfermedad arterial coronaria adquirida no sólo engloba la ateroesclerosis, sino que existen también numerosas entidades de carácter adquirido como aneurismas coronarios, pseudoaneurismas, disecciones, vasculitis y fístulas coronarias $^{(13)}$.

- Sospecha de anomalías congénitas coronarias. Presentes en aproximadamente el $1 \%$ de la población(14), y en ocasiones asociadas a la CoAo. Suponen un gran reto diagnóstico y su presencia debe tenerse en cuenta, en primer lugar, por los cardiólogos pediátricos y, secundariamente, por el cardiólogo especialista en cardiopatías congénitas, además de precisar un experto radiólogo habituado a la interpretación de este tipo de estudios. Existen multitud de anomalías congénitas del árbol coronario, algunas con diagnóstico precoz debido a su presentación clínica con sintomatología florida y típica, y otras cuya presentación atípica o paucisintomática, hace que finalmente sean diagnosticadas de forma casual en estudios realizados por otros motivos (a veces durante la propia evaluación de la CoAo). No todas las anomalías congénitas de las coronarias son peligrosas. Las técnicas de imagen hacen posible la distinción entre ellas, de forma que se pueda estimar el riesgo de muerte súbita, e indicar la actitud médica o quirúrgica más apropiada para tratarlas.

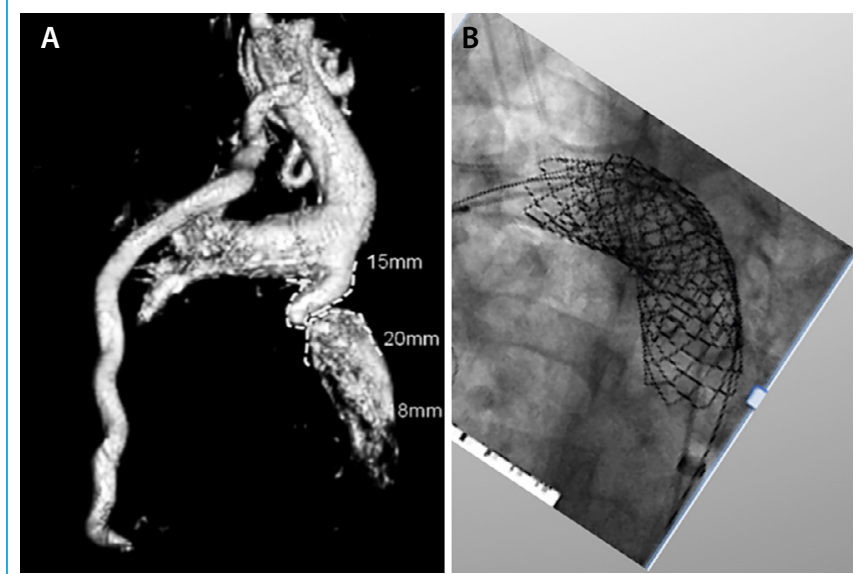

Figura 10. Planificación del tratamiento endovascular sobre una reconstrucción tridimensional con angiografía rotacional. Se valoran diámetros previos y posteriores de la coartación (A y B) y la longitud de la endoprótesis

\section{Neurorradiología en la coartación de aorta ${ }^{(5,6)}$}

Existe asociación entre la CoAo y la presencia de aneurismas intracraneales. La prevalencia de aneurismas en estos pacientes se estima en torno a un $10 \%$, suelen aparecer en la edad adulta y no existen predictores que permitan distinguir subgrupos de pacientes de alto riesgo en los que realizar cribado. El diagnóstico de aneurismas intracraneales puede realizarse tanto por RM cerebral como por TC cerebral o angio-TC cerebral. No existe ningún protocolo ni consenso sobre a qué pacientes ni cuándo realizar el cribado de aneurismas intracraneales, por lo que actualmente el estudio se indica en aquellos pacientes en los que exista sospecha de aneurismas intracraneales, exista clínica neurológica, o en cuadros agudos sugestivos de ACV. Podría plantearse la realización de un cribado de aneurismas intracraneales en pacientes jóvenes con CoAo, independientemente de si presentan otros factores de riesgo.

\section{Abreviaturas}

- BAV: válvula aórtica bicúspide

- CoAo: coartación de aorta

- ETT: ecocardiografía transtorácica

- HTA: hipertensión arterial

- HTP: hipertensión pulmonar

- Cardio-RM: resonancia magnética cardíaca

- TC: tomografía computarizada

\section{Ideas para recordar}

\section{¿Qué se le pide a la radiografía simple?}

- Primer estudio en la CoAo del adulto.

- Junto a la clínica permite sospechar la entidad, si existen signos radiológicos típicos.

- No es útil para toma decisiones ni seguimiento.

\section{¿Qué se le pide a la ETT?}

- Diagnóstico de CoAo nativa y seguimiento de CoAo reparada.

- Valoración funcional del ventrículo izquierdo y de complicaciones.

- Diagnóstico y cuantificación de lesiones y malformaciones asociadas. 


\section{¿Qué se le pide a la RM?}

- Valoración completa de la coartación, malformaciones asociadas y de la totalidad de la aorta, así como estudio y valoración de patología vascular en otras regiones (intracraneal).

- Valoración de la severidad.

- Planificación de la actitud terapéutica y seguimiento posterior.

- Valoración de fibrosis miocárdica y otras alteraciones del ventrículo.

\section{¿Qué se le pide a la TC?}

- Valoración completa de la coartación, malformaciones asociadas y de la totalidad de la aorta, así como estudio y valoración de patología vascular en otras regiones (intracraneal)

- Valoración de la anatomía coronaria, y la presencia de lesiones congénitas o adquiridas.

- Planificación de la actitud terapéutica y valoración de posibles complicaciones postintervención.

\section{Bibliografía}

1. Thakkar A, Chinnadurai P, Huei Lin C. Imaging adult patients with coarctation of the aorta. Current Opinion Cardiology 2017; 32: 503-512.

2. Dijkema EJ, Leiner T, Grotenhuis HB. Diagnosis, imaging and clinical management of aortic coarctation. Heart 2017; 103: 1148-1155.

3. Oliver JM, Gallego P, González A, et al. Risk factors for aortic complications in adults with coarctation of aorta. J Am Coll Cardiol 2004; 44: 1641-1647.
4. Krieger $\mathrm{E}$, Stout $\mathrm{K}$. The adult with repaired coarctation of the aorta. Heart 2010; 96: 1676-1681.

5. Mercado R, Lopez S, Cantu C, et al. Intracranial aneurysms associated with unsuspected aortic coarctation. J Neurosurgery 2002; 97 (5): 1221-1225.

6. Connolly HM, Huston J, Brown RD, et al. Intracranial aneurysms in patients with coarctation of the aorta: a prospective magnetic resonance angiographic study of 100 patients. Mayo Clin Proc 2003; 78 (12): 1491-1499.

7. Gibbs JL. Ultrasound and coarctation of the aorta. Br Heart J 1990; 64: 109-110.

8. Therrien J, Thorne SA, Wright A, et al. Repaired Coarctation: A "Cost-Effective" Approach to Identify Complications in Adults. JACC 20000; 35 (4): 997-1002.

9. Gacha P, Dabadiea A, Sorensena C, et al. Multimodality imaging of aortic coarctation: From the fetus to the adolescent. Diagnostic and Interventional Imaging 2016; 97: 581-590.

10. Secchi F, lozzelli A, Papini GDE, et al. MR imaging of aortic coarctation. Radiol med 2009; 114: 524-537.

11. Gacha P, Dabadiea A, Sorensena C, et al. Multimodality imaging of aortic coarctation: From the fetus to the adolescent. Diagnostic and Interventional Imaging 2016; 97: 581-590.

12. Montalescot G, Sechtem U, Achenbach S, et al. Guía de Práctica Clínica de la ESC 2013 sobre diagnóstico y tratamiento de la cardiopatía isquémica estable. Rev Esp Cardiol 2014; 67 (2): 135.e1-e81.

13. Dehaenea A, Jacquiera A, Falquea C, et al. Imaging of acquired coronary diseases: From children to adult. Diagnostic and Interventional Imaging 2016; 97: 571-580

14. Raimondi F, Bonnet D. Imaging of congenital anomalies of the coronary arteries. Diagnostic and Interventional Imaging 2016; 97: 561-569. 